\title{
A Templating Approach for Monodisperse Self-Assembled Organic Nanostructures
}

\author{
Steve R. Bull ${ }^{\dagger}$, Liam C. Palmer ${ }^{\dagger}$, Nathaniel J. Fry ${ }^{\dagger}$, Megan A. Greenfield ${ }^{\ddagger}$, Benjamin W. \\ Messmore ${ }^{\dagger}$, Thomas J. Meade ${ }^{\dagger}, \S$, and Samuel I. Stupp ${ }^{\dagger, \uparrow}$ \\ $\dagger$ Departments of Chemistry, Northwestern University, Evanston, Illinois 60208 \\ $\S$ Departments of Biochemistry and Molecular and Cellular Biology, Neurobiology and Physiology, and \\ Radiology, Northwestern University, Evanston, Illinois 60208 \\ $\$$ Departments of Chemical \& Biological Engineering, Northwestern University, Evanston, Illinois 60208 \\ IDepartment of Materials Science \& Engineering, and Feinberg School of Medicine, Northwestern \\ University, Evanston, Illinois 60208
}

The noncovalent self-assembly of molecules into supramolecular nanostructures presents exciting opportunities for complex structure and function inaccessible through step-by-step synthesis. ${ }^{1}$ In systems that generate supramolecular objects on the nano- and micrometer scales, it is desirable to control precisely nanostructure size in all dimensions. ${ }^{2}$ While precise structural control is known for closed spherical structures (e.g., micelles), the control of open structures is much more challenging. The tobacco mosaic virus (TMV) assembly represents an excellent example of templating by macromolecules. In that system, more than 2100 coat proteins spontaneously assemble into a 300-nm-long, rod-shaped structure around a single RNA molecule. The length of the assembly is determined by the length of the enclosed viral RNA template. ${ }^{3}$ The TMV coat proteins also self-assemble without RNA, affording structures that have the same diameter as native (untemplated) capsids but vary in length. The RNA template inhibits the unlimited self-assembly of the caspid proteins through specific interactions between constituent molecules. Herein, we describe an analogous strategy to control the length of a self-assembled nanofiber by using a rigid template.

The self-assembling molecule used in this study is peptide amphiphile (PA) 1, which consists of a charged amino acid sequence covalently attached to a hydrophobic alkyl chain (Figure 1).

4 These systems typically self-assemble into nanofibers as a result of hydrophobic collapse of the alkyl chains and $\beta$-sheet formation by the peptide segments. As in the TMV system without a template, PA monomers self-assemble into high-aspect-ratio nanofibers with uncontrollable lengths (micrometer scale) and well-defined diameters unless extensive bundling occurs among nanofibers. The average diameter of nanofibers formed by $\mathbf{1}$ was measured by atomic force microscopy (AFM) to be $5.3 \pm 0.6 \mathrm{~nm}$ (Figure 2a) and is in good agreement with the width determined by transmission electron microscopy (TEM).

We designed and synthesized template molecule 2 that contains a hydrophobic oligo( $p$ phenylene ethynylene) core with bulky hydrophilic end caps in the shape of a dumbbell. ${ }^{5-7}$ The hydrophobic rigid-rod defines a precise length that can be encapsulated within the hydrophobic core of the PA nanofibers. We hypothesized that in an aqueous solution, PA $\mathbf{1}$ and template $\mathbf{2}$ would co-aggregate through hydrophobic collapse. ${ }^{8}$ We also assume that the

E-mail: s-stupp@northwestern.edu. 
hydrophilic polyethyleneglycol (PEG) caps at each end of the rigid rod would disrupt the onedimensional fiber assembly (Figure 1c,d).

When 2 alone was drop-cast from aqueous solution on mica, AFM showed ribbon-like supramolecular structures (see Supporting Information). We believe that these dumbbell molecules aggregate upon drying on the hydrophilic substrate, maximizing $\pi-\pi$ stacking interactions between rigid-rods. Similar self-assembly has been reported previously with a related oligo( $p$-phenylene) dumbbell-shaped molecule. ${ }^{5}$

Mixtures of 1 and 2 were studied using molar ratios of 200:1, 500:1, and 1000:1. AFM images of the 200:1 mixture of $\mathbf{1}$ and $\mathbf{2}$ revealed small aggregates (Figure 2b). The AFM of the 200:1 mixture appears completely different from the control sample of $\mathbf{1}$ alones $\mathbf{- 1}$ and $\mathbf{2}$ interact to form small structures rather than high-aspect-ratio nanofibers. The 200:1 solution of aggregates has heights of $5.5 \pm 0.7 \mathrm{~nm}$ by AFM, comparable to fiber heights of $5.3 \pm 0.6 \mathrm{~nm}$. In mixed samples with a higher molar fraction of PA 1 (500:1 or 1000:1), we observe both small micellelike nanostructures and longer nanofibers, indicating that there is not enough of template $\mathbf{2}$ to fully suppress the extensive one-dimensional assembly of $\mathbf{1}$ (see Supporting Information).

The fiber widths from 1 were determined by TEM to be $5.1 \pm 0.7 \mathrm{~nm}$ (see Supporting Information). As in the AFM images, the TEM images of the 200:1 mixture show micelle-like structures with diameters of approximately $5.6 \pm 0.6 \mathrm{~nm}$. We attribute the appearance of these round structures to an end-on view of the aggregate. These data indicate that the $\mathbf{1 . 2}$ aggregate has a diameter similar to that of nanofibers formed by PA molecules alone, suggesting the template molecules can control the length of open, nonspherical supramolecular structures.

To better understand the structure of the 200:1 assembly without the effects of drying, we prepared additional TEM samples according to a quick-freeze deep etch (QFDE) protocol. Since the sample is prepared by subliming ice from a frozen solution, we expect the small aggregates to appear in a variety of orientations. Indeed, we observed slightly elongated nanostructures that we can estimate to have dimensions of $\sim 8.8 \mathrm{~nm}$ diameter and $14.0 \mathrm{~nm}$ length (Figure 3). TEM images are taken from replica prepared by sputter-coating $\sim 1.2 \mathrm{~nm}$ of platinum onto the sample, so the measured lengths and widths should be $\sim 2.4 \mathrm{~nm}$ greater than the actual size. In fact, we observe that the small aggregates display an aspect ratio consistent with the dimensions of our proposed mechanism for templated self-assembly $(\sim 5.5 \mathrm{~nm} \times 12.2$ $\mathrm{nm})$.

Dynamic light scattering (DLS) was used to characterize the effect of the dumbbell template on PA assembly in solution. A change in the self-assembled structures from long fibers to small aggregates should result in a measurable change in hydrodynamic radius. DLS measurements on solutions of 1 confirmed the presence of large structures ( $>1 \mu \mathrm{m}$, see Supporting

Information). While 2 was observed to form ribbon-like structures by AFM as a film on mica (attributed to drying effects), DLS indicates that, in solution, $\mathbf{2}$ has a hydrodynamic radius of $\sim 17.4 \mathrm{~nm}$. The mixed sample consisting of a 200:1 molar ratio gave a size distribution centered at $\sim 17.9 \mathrm{~nm}$. We do not expect a significant change in hydrodynamic radius of the dumbbell molecules with and without the PA, since the long axis of the assembly is the same as that of the template. In the mixed samples of 500:1 and 1000:1 (consistent with the microscopy data), we observe scattering from large structures that suppresses any signal from smaller species, indicating the persistence of fibers. Circular dichroism (CD) revealed the presence of $\beta$-sheet structure in solutions of $\mathbf{1}$ alone and in the 200:1 mixture, confirming that the PA molecules were still highly ordered in the $\mathbf{1 \cdot 2}$ aggregate. While $\mathbf{2}$ limits the ability of $\mathbf{1}$ to form extended supramolecular structures, the intermolecular basis of PA self-assembly remains unchanged. At such a low concentration of $\mathbf{2}$, we expect the PA to dominate the spectrum, but we cannot exclude the possibility of chirooptic activity from the oligo-(phenylene ethynylene). ${ }^{9}$ The 
fluorescence spectrum of $\mathbf{2}$ remains unchanged after co-assembly with $\mathbf{1}$, as we would expect for a molecule with a small dipole. While it is difficult to prove direct interaction between $\mathbf{1}$ and $\mathbf{2}$ spectroscopically, we believe that the microscopy and light scattering data provide compelling evidence for a templated change in the self-assembly behavior.

We conclude that the formation of nonspherical supramolecular aggregates with controlled dimensions has been realized using a rigid-rod dumbbell-shaped template that limits the onedimensional self-assembly of PA molecules. AFM and TEM data show a dramatic change in the form of the PA supramolecular aggregate upon addition of the dumbbell template from high-aspect-ratio nanofibers to small, nearly monodisperse nanostructures. DLS and CD confirm this behavior in solution. This general strategy may provide control over properties of supramolecular aggregates that are sensitive to dimension and may offer more precise control of their stoichiometric composition. The latter has potential to control the delivery of therapeutic molecules in supramolecular form. Finally, shape control within the molecular template may lead to greater complexity in the supramolecular nanostructure.

\section{Supplementary Material}

Refer to Web version on PubMed Central for supplementary material.

\section{Acknowledgment}

The authors thank Northwestern University's NIH Biotechnology Training Program (predoctoral fellowship to S.R.B.), the Department of Homeland Security (predoctoral fellowship to M.A.G.), the National Science Foundation, the Department of Energy, and the National Institutes of Health (5R01EB005866-02 and CA119341-02) for financial support. This work made use of NUANCE (TEM, AFM), Biological Imaging Facility (TEM), IMSERC (NMR, MS), and Keck Biophysics Facility (CD).

\section{References}

(1)(a). Lehn JM. Angew. Chem., Int. Ed. Engl 1988;27:89-112. (b) Whitesides GM, Grzybowski B. Science 2002;295:2418-2421. [PubMed: 11923529] (c) Palmer LC, Velichko YS, Olvera de la Cruz M, Stupp SI. Philos. Trans. R. Soc., Ser. A 2007;365:1417-1433. (d) Brunsveld L, Folmer BJB, Meijer EW, Sijbesma RP. Chem. Rev 2001;101:4071-4097. [PubMed: 11740927] (e) Shimizu T, Masuda M, Minamikawa H. Chem. Rev 2005;105:1401-1443. [PubMed: 15826016] (f) Yamamoto Y, Fukushima T, Suna Y, Ishii N, Saeki A, Seki S, Tagawa S, Taniguchi M, Kawai T, Aida T. Science 2006;314:1761-1764. [PubMed: 17170300]

(2). Hunter CA, Tomas S. J. Am. Chem. Soc 2006;128:8975-8979. [PubMed: 16819894]

(3). Klug A. Philos. Trans. R. Soc. London, Ser. B 1999;354:531-535. [PubMed: 10212932]

(4)(a). Hartgerink JD, Beniash E, Stupp SI. Science 2001;294:1684-1688. [PubMed: 11721046] (b) Hartgerink JD, Beniash E, Stupp SI. Proc. Natl. Acad. Sci. U.S.A 2002;99:5133-5138. [PubMed: 11929981] (c) Paramonov SE, Jun HW, Hartgerink JD. J. Am. Chem. Soc 2006;128:7291-7298. [PubMed: 16734483] (d) Jiang HZ, Guler MO, Stupp SI. Soft Matter 2007;3:454-462.

(5). Bae J, Choi JH, Yoo YS, Oh NK, Kim BS, Lee M. J. Am. Chem. Soc 2005;127:9668-9669. [PubMed: 15998054]

(6). Gin MS, Yokozawa T, Prince RB, Moore JS. J. Am. Chem. Soc 1999;121:2643-2644.

(7). Xue CH, Luo FT. Tetrahedron 2004;60:6285-6294.

(8). Based on our pRevious work with a similar but shorter oligophenylene dumbbell, we concluded that this was the minimum length to observe templation.

(9). Wilson JN, Steffen W, McKenzie TG, Lieser G, Oda M, Neher D, Bunz UHF. J. Am. Chem. Soc 2002;124:6830-6831. [PubMed: 12059196] 


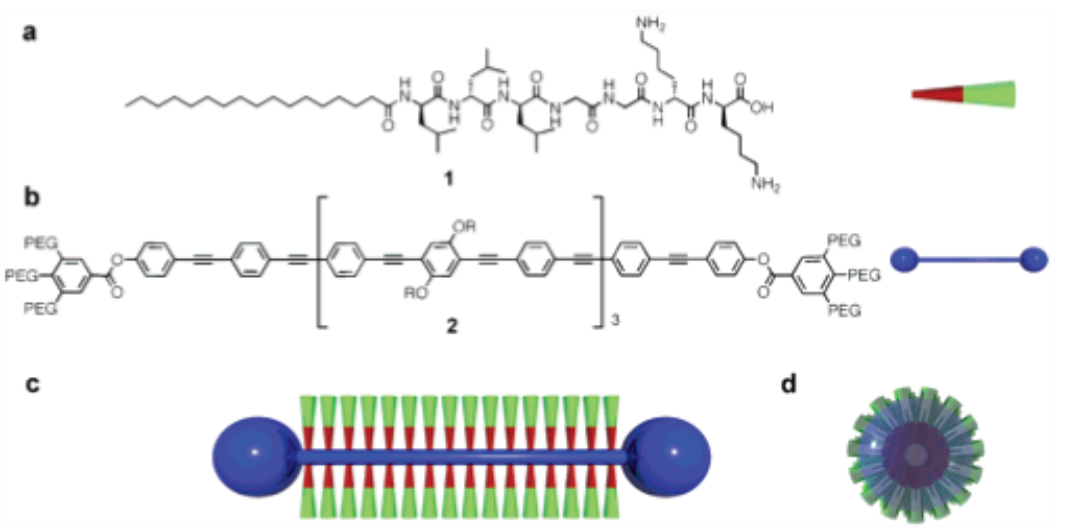

Figure 1.

(a) Peptide amphiphile monomer 1 and a cartoon representation. (b) Oligo(phenylene ethynylene) dumbbell 2 ( $\mathrm{R}=2$-octyldodecyl, $\mathrm{PEG}=\left(\mathrm{OCH}_{2} \mathrm{CH}_{2}\right)_{n} \mathrm{OCH}_{3}$, where $\left.n=7.4\right)$ and cartoon representation. (c,d) Cartoon representation of the PA self-assembling around the dumbbell molecule creating a structure of discrete length. 


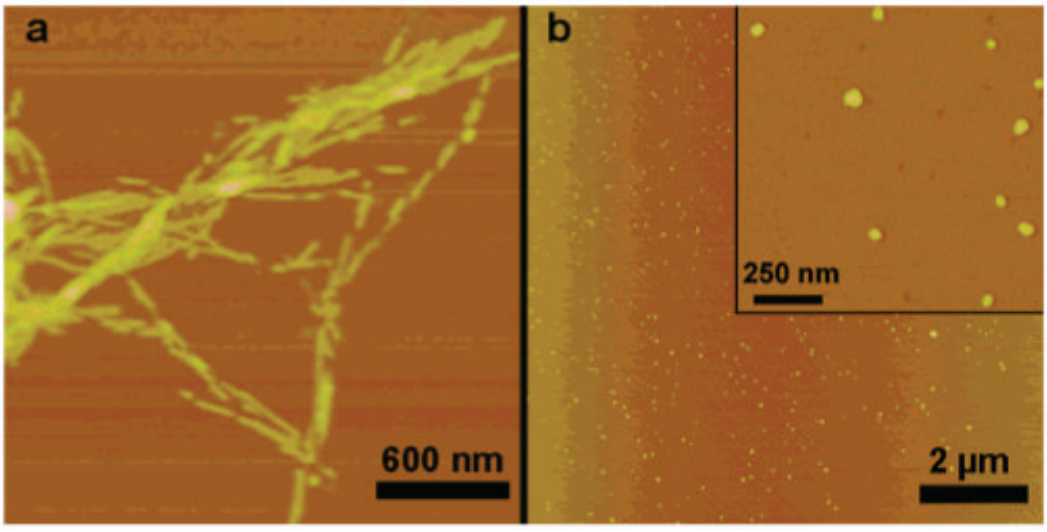

Figure 2.

AFM height images of aqueous solutions drop-cast on mica ( $1 \mathrm{mg} / \mathrm{mL}$ of 1). (a) Peptide amphiphile 1 alone gave fibers with heights of $5.3 \pm 0.6 \mathrm{~nm}$. (b) A 200:1 molar ratio mixture of $\mathbf{1}$ and $\mathbf{2}$ showed no fibers in a wide-field view. 


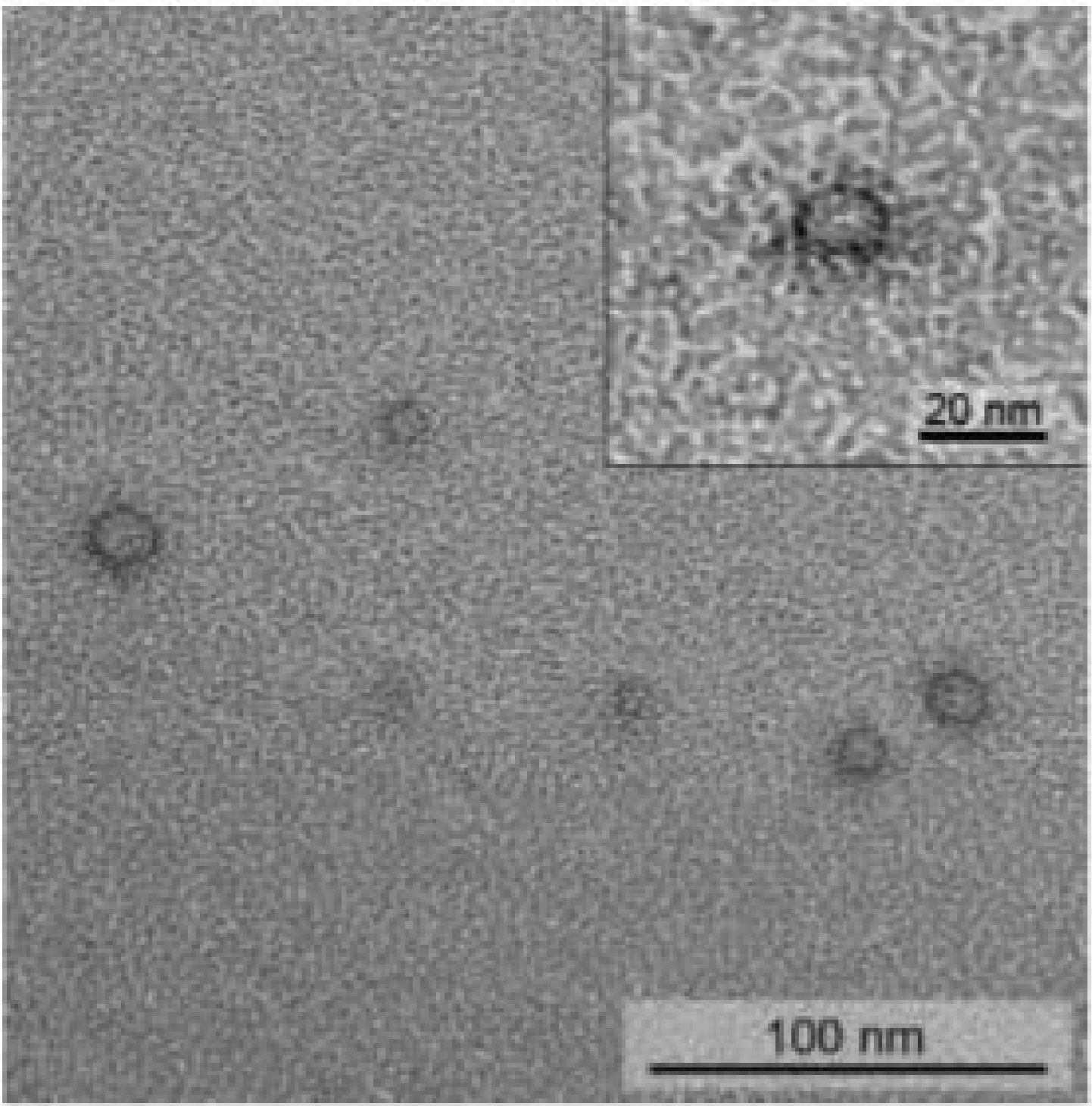

Figure 3.

QFDE TEM of 200:1 molar ratio mixture of $\mathbf{1}$ to $\mathbf{2}$, revealing the aspect ratio of the small aggregates. Inset: The length and width are in agreement with the size expected for the templated assembly. The dark exteriors in the replica result from the deposition of the platinum. 